\title{
Recuperação do Relevo do Embasamento de uma Bacia Sedimentar através da criação de Modelo de Resistividade 1D Composto para Região Central da Bacia do Paraná, Centro-sul do Brasil.
}

\author{
Carreira, V. R. 1, La Terra, E. F. 2, Fontes, S. L. 3 ; 1, 2, 3 Observatório Nacional, Rio de Janeiro, Brasil.
}

Copyright 2016, SBGf - Sociedade Brasileira de Geofísica.

Este texto foi preparado para a apresentação no VII Simpósio Brasileiro de Geofísica, Ouro Preto, 25 a 27 de outubro de 2016. Seu conteúdo foi revisado pelo Comitê Técnico do VII SimBGf, mas não necessariamente representa a opinião da SBGf ou de seus associados. É proibida a reprodução total ou parcial deste material para propésitos comerciais sem prévia autorizaç ão da SBGf.

\section{Abstract}

This paper aims to define the limits between the top layer of the basement and the bottom layer of the Sedimentary Basin of the Paraná Basin by magnetotelluric data and its dimensional analysis, complementing the magnetotelluric studies that are being promoted by the National Petroleum Agency ANP. The studies of large structures the basement of sedimentary basins are of paramount importance for the understanding of of its tectonic-stratigraphic evolution. The Paraná Basin, in particular, is a shallow basin with estimated depocenter $7000 \mathrm{~m}$. Hypothetical test indicates a good recovered synthetic basementbasin limit. The real data shows a limit basement-basin around $5.5 \mathrm{Km}$ in its highest depth in concordance with well data and previous studies.

\section{Introdução}

A Bacia sedimentar do Paraná está localizada no centrosul da América do Sul (Fig. 1) e engloba uma área de aproximadamente $1.100 .000 \mathrm{Km}^{2}$, dentro do território brasileiro. Distribui-se ainda pela República do Paraguai, Uruguai e Argentina, por cerca de $100.000 \mathrm{Km}^{2}$ (Zalan and Wolf, 1987).

Caracteriza-se como uma bacia do tipo intraplataformal, localizada sob uma litosfera rígida, do tipo continental e possui formado ovalado (Cordani et al., 1984). Sua história evolutiva teve início no Cambriano e insere-se em um contexto de bacia cratônica marginal, sob domínio flexural de crosta (Borghi, 2002).

Apresenta a profundidade de cerca de 7000 metros, no depocentro, e é preenchida por um pacote de rochas sedimentares do Paleozóico e do Mesozóico. Derrames de lavas deram origem as rochas basálticas do Cretáceo cobrindo boa parte da superfície da bacia, chegam a espessuras de 1700 metros. As intrusivas estão presentes na bacia, marcadas por diques e sills, também do Cretáceo. Os diques formam um cinturão de afloramentos que bordejam a bacia nas seções Nordeste, Leste, Sul e Oeste e cobrem boa parte da superfície (Milani et al., 2007) apud (Borghi, 2002).

A Bacia do Paraná desenvolveu-se sobre uma área de escudo do continente Gondwana Sul e é composta por uma série de núcleos cratônicos, rodeados por vários cinturões móveis e cobertos por bacias molássicas, que foram desenvolvidas durante o ciclo termo-tectônico Brasiliano que se estendeu desde o neoproterozóico até - Ordoviciano. A deformação decorrente deste ciclo teve início entre $700 \mathrm{Ma}$ e $650 \mathrm{Ma}$, sendo que a maior parte das intrusões de granitos que podemos observar na Bacia, situou-se dentro do limite entre o Proterozóico e o Paleozóico (cerca de $570 \mathrm{Ma}$ ) com resfriamento durante o Cambro-Ordoviciano entre 500-450 Ma (Zalan and Wolf, 1987; Hawkesworth et al., 2000).

O embasamento que circunda a Bacia do Paraná é dividido em: margem Leste/Sudeste, representado pelas faixas Dom Feliciano e Ribeira, de idade Brasiliana e de direção NE-SW, separados por um núcleo cratônico designado Rio de La Plata/ Luiz Alves; margem Norte/Nordeste, representada pela faixa Uruaçu, de idade mesoproterozóica, de direção NW e por dois maciços arqueanos (Guaxupé e Goiás) remobilizados durante o ciclo Brasiliano; margem Oeste/Noroeste representada pela faixa de dobramentos Paraguai/Araguaia, também do ciclo Brasiliano, que delimita o extremo da borda Noroeste da Bacia (Borghi, 2002; Hawkesworth et al., 2000).

O registro estratigráfico da Bacia do Paraná é formado por pacote sedimentar e magmático de espessura máxima em torno de $7000 \mathrm{~m}$, que coincide geograficamente com o depocentro estrutural da sinéclise e com a calha do rio paraná (Milani and Ramos, 1998). O registro estratigráfico da Bacia do Paraná é dividido em seis unidades de ampla escala ou supersequências (Vail et al., 1977) na forma de pacotes rochosos com intervalos temporais de algumas dezenas de milhões de anos de duração e envelopados por superfícies de discordância de caráter inter-regional: Rio Ivaí (Ordoviciano-Siluriano), Paraná (Devoniano), Gondwana I (Carbonífero-Eotriássico), Gondwana II (Meso a Neotriássico), Gondwana III (Neojurássico-Eocretáceo) e Bauru (Neocretáceo). As três primeiras supersequências são representadas por sucessões sedimentares que definem ciclos transgressivos e regressivos ligados às oscilações do nível relativo do mar, durante o Paleozóico, ao passo que as demais correspondem a pacotes de sedimentos continentais com rochas ígneas associadas. As unidades formais da litoestratigrafia, quais sejam os grupos, formações e membros comumente utilizados na descrição do arranjo espacial dos estratos da bacia, inserem-se como elementos particularizados neste arcabouço aloestratigráfico de escala regional (Milani et al., 2007).

O mapa geológico-estrutural (Fig. 1) apresenta as formações aflorantes bem como as principais lineamentos e o Arco de Ponta Grossa (Vidotti et al., 1998; Bizzi et al., 2003). 


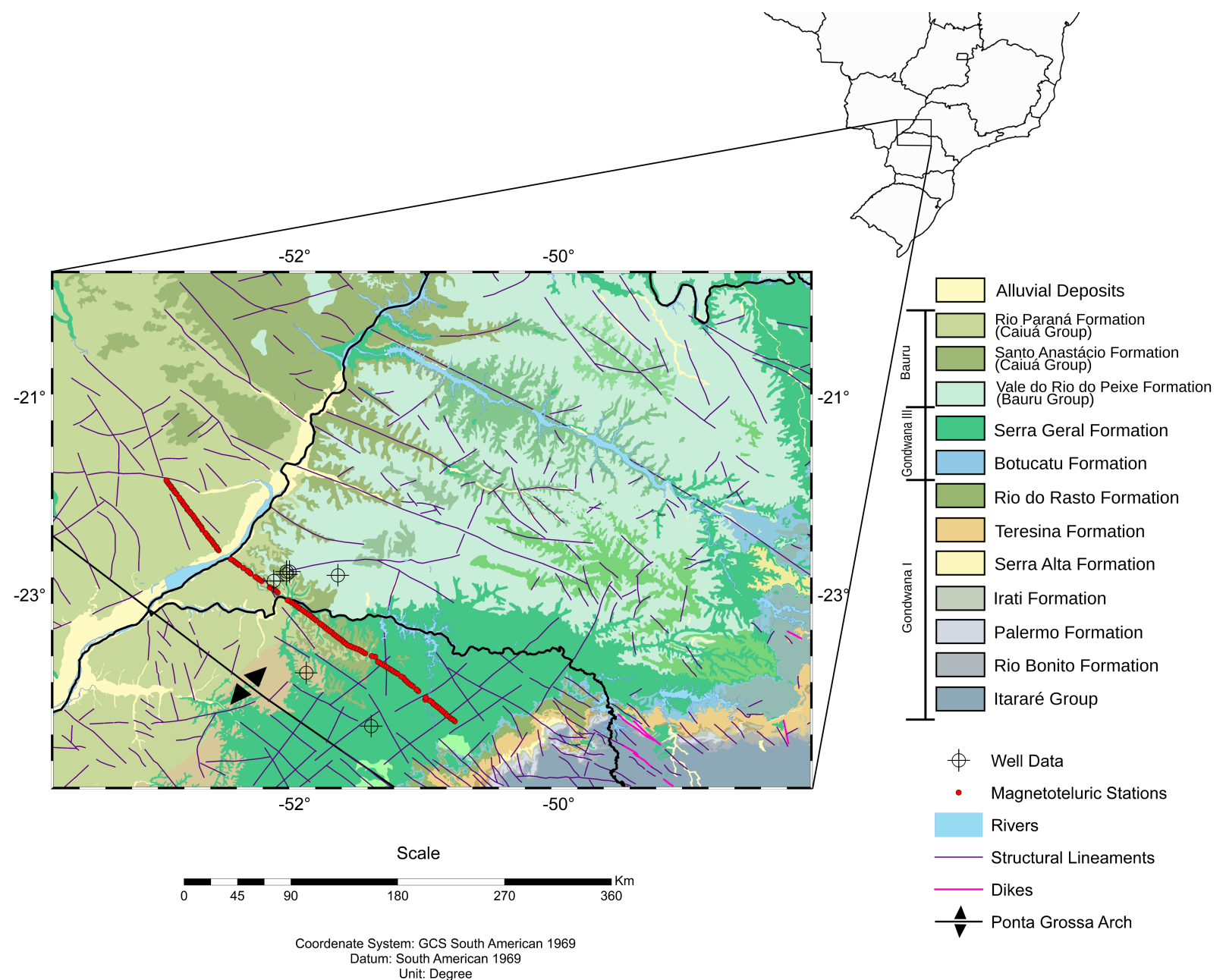

Figure 1: Mapa geológico e de localização da área de estudo.

A modelagem e inversão geofísica de resistividade 1D foi empregada para delimitar o relevo do embasamento.

Como teste da resposta da metodologia de inversão 1D de Occam (Constable et al., 1987) a um embasamento complexo, um modelo sintético de resistividade foi proposto para uma bacia tipo sinéclise com embasamento contendo Horsts e Grabens.

A região de estudo (Fig. 1) ocupa a porção central da Bacia do Paraná, entre os paralelos geográficos $21^{\circ}$ e $24^{\circ}$ $\mathrm{S}, 54^{\circ}$ e $50^{\circ} \mathrm{W}$, entre os estados do Mato Grosso do Sul, São Paulo e Paraná. Os dados magnetotelúricos foram gerados por levantamento terrestre adquiridos pela ANP e acompanhados pelo Observatório Nacional.

\section{Metodologia}

A metodologia adotada neste trabalho baseia-se na análise de dimensionalidade do dado magnetotelúrico cujos invariantes rotacionais possuem comportamento peculiar dependendo da situação geológica a onda eletromagnética se encontra (Vozoff, 1986; Fiona and Bahr, 2005; Figueiredo, 2008).

Os dados utilizados neste trabalho são compostos por 150 estações magnetotelúricas espaçadas a cada $2 \mathrm{~km}$. Este perfil estende-se por $300 \mathrm{Km}$ e possui orientação de $130^{\circ}$ com o norte verdadeiro. Balizou-se o resultado final com dados de 6 poços estratigráficos e com estudo geológico prévio da área de estudo.

A rotina de processamento robusto seguiu a metodologia proposta por Egbert and Booker (1986). Uma vez processados os dados foram corrigidos para o seu real valor de resistividade pelo procedimento de correção estática (Gamble et al., 1979; Meju, 1994; Árnason, 2008).

Uma vez corrigidos e determinada a dimensionalidade foi possível criar um modelo inverso somente da parte do dado correspondente a parte 1D do dado que representa a Bacia Sedimentar (Word et al., 1970; Constable et al., 1987).

Para que fosse possível inferir a potencialidade da metodologia proposta foi realizado um teste de hipótese cujo modelo representa a realidade da área estudada, incluso nele, estão representados os altos e os baixos estruturais do embasamento sintético bem como a bacia sedimentar sintética.

\section{O Tensor de Impedância}



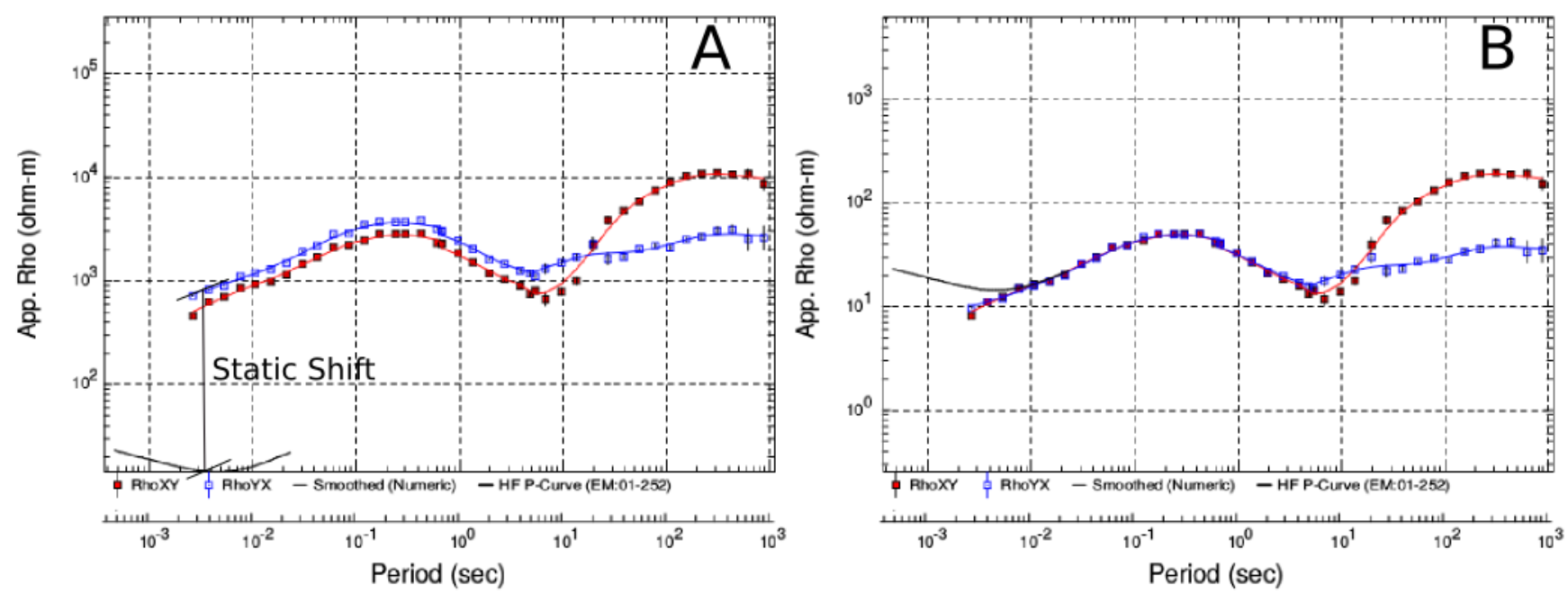

Figure 2: Deslocamento vertical da curva de resistividade aparente para estação 252 (A). Curva de resistividade aparente após a correção galvânica da estação ON-252 (B).

O método magnetotelúrico é um método não-invasivo no domínio da frequência capaz de estimar o contraste de resistividade em subsuperfície a partir de medidas simultâneas na superfície do terreno dos campos elétricos e magnéticos naturais. Realiza investigações desde a superfície do terreno até pouco mais de uma centena de quilômetros em profundidade.

A fonte de sinal do método envolve a medição da variação natural dos campos elétricos e magnéticos em subsuperfície da Terra, resultantes, principalmente, de tempestades elétricas, na baixa atmosfera, que são a causa dominante dos campos eletromagnéticos entre $1 \mathrm{~Hz}$ e $10 \mathrm{kHz}$, Contudo, os campos eletromagnéticos abaixo de $1 \mathrm{~Hz}$ se originam primariamente na magnetosfera terrestre (Figueiredo, 1997, 2008)

O princípio teórico é fundamentado na teoria eletromagnética, e, nas equações de Maxwell. Afim de se considerar o fenômeno de indução eletromagnética na Terra, são necessárias algumas considerações matemáticas, dentre as quais: o planeta Terra não gera energia eletromagnética, somente a dissipa ou a absorve, os campos conservativos e afastados de suas fontes, a fonte principal do sinal EM é gerada na ionosfera, não há acúmulos de cargas livres dentro da Terra em camadas (em uma Terra multi-dimensional as cargas podem ser acumuladas ao longo das descontinuidades. Isto é a fonte de geração de um fenômeno não-indutivo conhecido como static shift), a corrente de deslocamento é considerada nula, e o tratamento da indução eletromagnética, na Terra, puramente através de processos de difusão (Fiona and Bahr, 2005).

Os elementos do tensor de impedância são calculados no domínio da frequência $Z(\omega)$ entre as componentes horizontais dos campos elétricos e magnéticos incidentes induzidos, que são dados por uma matriz magnetotelúrica $2 \times 2$ de um tensor de impedância complexo (Jones, 1988).

$$
E(\omega)=Z(\omega) H(\omega)
$$

Na forma matricial, Eq. 2;

$$
\left[\begin{array}{l}
E_{x}(\omega) \\
E_{y}(\omega)
\end{array}\right]=\left[\begin{array}{ll}
Z_{x x}(\omega) & Z_{x y}(\omega) \\
Z_{y x}(\omega) & Z_{y y}(\omega)
\end{array}\right] \cdot\left[\begin{array}{l}
H_{x}(\omega) \\
H_{y}(\omega)
\end{array}\right]
$$

A partir de $Z(\omega)$ obtém-se a resistividade aparente $\rho_{a}$, Eq. 3 , e da fase, $\phi$, Eq. 3 (Vozoff, 1986).

$$
\begin{gathered}
\rho_{a, i j}=\frac{1}{\omega \mu_{0}}\left|Z_{i j(\omega)}\right|^{2} \\
\phi_{i j}=\tan ^{-1}\left(\frac{\operatorname{Im}\left(Z_{i j(\omega)}\right)}{\operatorname{Re}\left(Z_{i j(\omega)}\right)}\right)
\end{gathered}
$$

\section{Correção Estática}

Devido a distorção galvânica provocada pelas heterogeneidades, ou corpos 3D próximos à superfície (Sternberg et al., 1988; Árnason, 2008; Mwakirani et al., 2012) foi necessário realizar a correção estática nas curvas de resistividade aparente com uso das curvas de resistividade TEM (Transient Electromagnetic) (Gamble et al., 1979; Meju, 1994; Árnason, 2008).

A Fig. 2 mostra o deslocamento por um fator $S$, na curva de resistividade aparente ao comparado com o dado TEM, para estação ON-252.

\section{Análise de Dimensionalidade}

Os resultados MT apresentaram situações cuja dimensionalidade variou de 1D, 2D a 3D (Fig. 3).

Para uma Terra 1D a condutividade varia apenas com profundidade. Em uma terra $2 \mathrm{D}$ a variação da condutividade ocorre ao longo de uma das direções horizontais e na vertical. $O$ caso $1 \mathrm{D}$ e $2 \mathrm{D}$ assumem respectivamente os seguintes valores (Figueiredo, 2008; Fiona and Bahr, 2005). 


$$
1 D\left\{\begin{array} { l } 
{ Z _ { x x } ( \omega ) = Z _ { y y } ( \omega ) = 0 } \\
{ Z _ { x y } ( \omega ) = - Z _ { y x } ( \omega ) }
\end{array} 2 D \left\{\begin{array}{l}
Z_{x x}(\omega)=-Z_{y y}(\omega)=0 \\
Z_{x y}(\omega) \neq-Z_{y x}(\omega)
\end{array}\right.\right.
$$

No caso $3 D$ os invariantes são todos diferentes de zero (Figueiredo, 2008).

Foram utilizados parâmetros clássicos simples para análise da dimensionalidade como o Tipper e a Elipticidade.

A elipticidade varia de acordo com a amplitude e direção das componentes do tensor de impedância, e, pode ser descrita de acordo com a Eq. 3 (Vozoff, 1986; Fiona and Bahr, 2005).

$$
\beta(\omega)=\frac{Z_{x x}(\omega)-Z_{y y}(\omega)}{Z_{x y}(\omega)+Z_{y x}(\omega)}
$$

Onde os termos $Z_{x x}(\omega)-Z_{y y}(\omega)$ e $Z_{x y}(\omega)+Z_{y x}(\omega)$ são constantes invariantes do tensor de impedância (Figueiredo, 2008).

Em uma realidade 1D, esta propriedade é zero para um dado totalmente livre de ruído (Vozoff, 1986). A Fig. 3 ilustra a análise da elipticidade da estação ON-252.

Pode-se considerar $H_{z} \approx 0$, nos casos, onde não se tem uma variação lateral. (Vozoff, 1986; Fiona and Bahr, 2005) A relação entre $H_{z}$ e o campo magnético horizontal, em qualquer frequência, pode ser escrito de acordo com a Eq. 4.

$$
H_{z}=T_{x} H_{x}+T_{y} H_{y}
$$

Como em uma Terra 2D com o strike na direção $x^{\prime}$, a Eq. 4, pode ser reescrita segundo a Eq. 5.

$$
H_{z}=T_{y}^{\prime} H_{y}
$$

Onde $T^{\prime}$, é a inclinação do vetor $H$, em relação a um plano horizontal, também chamada de Tipper. No caso uma situação 1D o valor do Tipper será igual a zero para um dado sem ruído (Vozoff, 1986).

Como os dados reais não são livres de ruído, adotou-se para o presente trabalho valores de Tipper próximos a zero como sendo característicos de uma realidade 1D. A mudança de dimensionalidade no caso 2D ou 3D se dá na quebra da curva do Tipper, conforme mostra a Fig. 3. Nota-se que quando as componentes $X Y$ e $Y X$ são iguais temos identificado uma realidade unidimensional para 0 dado.

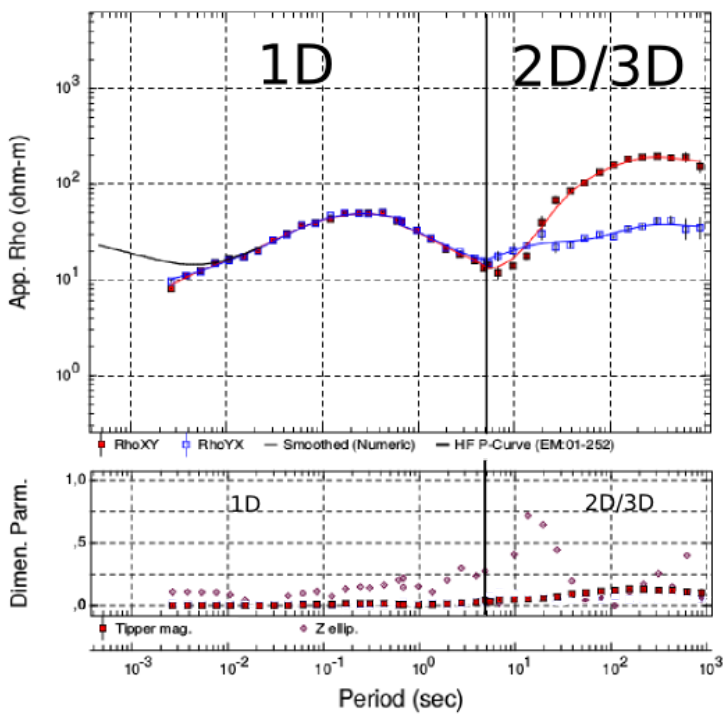

Figure 3: Parâmetros de dimensionalidade da estação ON252. Os pontos em vermelho são a a magnitude do Tipper e os círculos representam a elipsidade.

\section{Modelagem e Inversão $1 D$}

Na modelagem direta magnetotelúrica, considera-se uma Terra 1D como possuindo $n$-camadas homogêneas e isotrópicas (Figueiredo, 1997). Para cada jesima camada a resistividade $\rho_{j}$ e a espessura $h_{j}$ definem uma impedância intrínseca $I_{j}=\sqrt{k_{j} / \rho_{j}}$, onde $k_{j}=\sqrt{i \omega \mu \sigma_{j}}$ é o número de onda (Meju, 1994).

$$
\mathrm{Z}_{\mathrm{j}}=\mathrm{I}_{\mathrm{j}} \frac{1-\mathrm{R}_{\mathrm{j}} \mathrm{E}_{\mathrm{j}}}{1+\mathrm{R}_{\mathrm{j}} \mathrm{E}_{\mathrm{j}}}, \mathrm{j}=\mathrm{n}-1, \ldots, 1
$$

Onde $R$ é o coeficiente de reflexão, e, pode ser descrito de acordo com a Eq. 7.

$$
R_{j}=\frac{I_{j}-Z_{j+1}}{I_{j}+Z_{j+1}}
$$

Onde $Z_{n}=I_{n}$. Sendo a aplicação da forma recursiva da Eq. 6, partindo do fundo da camada, onde $j=n-1$. Desta forma, a impedância é obtida da camada inferior, para a camada mais superficial. A impedância EM medida na superfície da terra 1D é descrita de acordo com a Eq. 8 (Word et al., 1970).

$$
Z_{j}=\frac{k_{j}}{\sigma_{j}} \frac{k_{j}\left(1-E_{j}\right)+Z_{j+1}\left(1+E_{j}\right)}{k_{j}\left(1+E_{j}\right)+Z_{j+1}\left(1-E_{j}\right)}
$$

Para propósitos de modelagem é usual utilizar a resistividade aparente e a fase de Cargniard. Representados, respectivamente, pelas Eq. 9 e Eq. 10.

$$
\rho_{a}=\frac{1}{\omega \mu}\left|Z_{1}\right|^{2}
$$




$$
\phi=\tan ^{-1}\left(\frac{\operatorname{Im}\left(Z_{1}\right)}{\operatorname{Re}\left(Z_{1}\right)}\right)
$$

No método MT, a Terra é parametrizada em uma sucessão de camadas de resistividades $\rho_{i}$, e, espessuras $h_{i}$, utilizando-se em geral na inversão do logaritmo destas grandezas, pois a resistividade aparente mostra uma distribuição norma logarítmica (Figueiredo, 1997).

A metodologia usada na inversão 1D MT é denominada Occam. Este procura o modelo mais suave, que ajuste os dados dentro de um certo erro. Para tal, impõese um vínculo na minimização dos erros quadráticos em termos de aspereza do modelo, o inverso da suavidade, a rugosidade, que pode ser descrito de acordo com a Eq. 11 e a Eq. 12 (Constable et al., 1987).

$$
R_{1}=\int\left(\frac{d m}{d z}\right)^{2} d z
$$

$\mathrm{Ou}$

$$
R_{2}=\int\left(\frac{d^{2} m}{d z^{2}}\right)^{2} d z
$$

Desta forma o modelo suave esperado pode ser descrito de acordo com a Eq. 13.

$\mathbf{m}^{k+1}=\left[(\mathbf{W A})^{T}(\mathbf{W A})+\Gamma \mathbf{D}^{T} \mathbf{D}\right]^{-1}(\mathbf{W A})^{T}\left[(\mathbf{W y})+(\mathbf{W A}) \mathbf{m}^{k}\right]$

Onde o parâmetro do modelo $\mathbf{m}$, para o MT, é a resistividade, $\mathbf{m}^{k+1}$ é a correção no vetor $\mathbf{m}$ na $K_{\text {esima }}$ iteração, D é a matriz do operador diferença, ou, a matriz suavidade. Na metodologia Occam procura o modelo mais suave, que ajusta os dados dentro de um certo erro, A é a matriz sensibilidade, $\mathbf{W}$ é a matriz pesos, $\Gamma$ parâmetro de regularização (Word et al., 1970; Constable et al., 1987; Figueiredo, 1997).

\section{Resultados}

\section{Dado Sintético}

Para modelagem do topo do embasamento com dados MT foi gerado um modelo de camadas cuja variação da resistividade se dá somente na direção vertical com o objetivo de correlacionar o comportamento estratigráfico horizontal de uma bacia sedimentar.

$O$ teste de hipótese que discretiza o relevo do embasamento foi realizado através da modelagem direta e inversa de em uma seção $2 \mathrm{D}$ representando bacia do tipo sinéclise, sendo a que mais se aproxima da geologia da região estudada.

Uma malha de células com diferentes valores de resistividade representando a seção geofísica foi elaborada a partir do modelo geológico. O modelo geofísico (Fig. 4) é composto de três camadas que representam as rochas sedimentares da bacia, onde se atribuiu diferentes valores de resistividade, entre 200 a

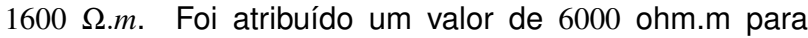
o embasamento. Um relevo rugoso representando o relevo do embasamento foi criado simulando estruturas geológicas de Horsts e Grabens. Um ruído aleatório gaussiano de $2.5 \%$ foi somado ao dado (Geosystem, 2011).

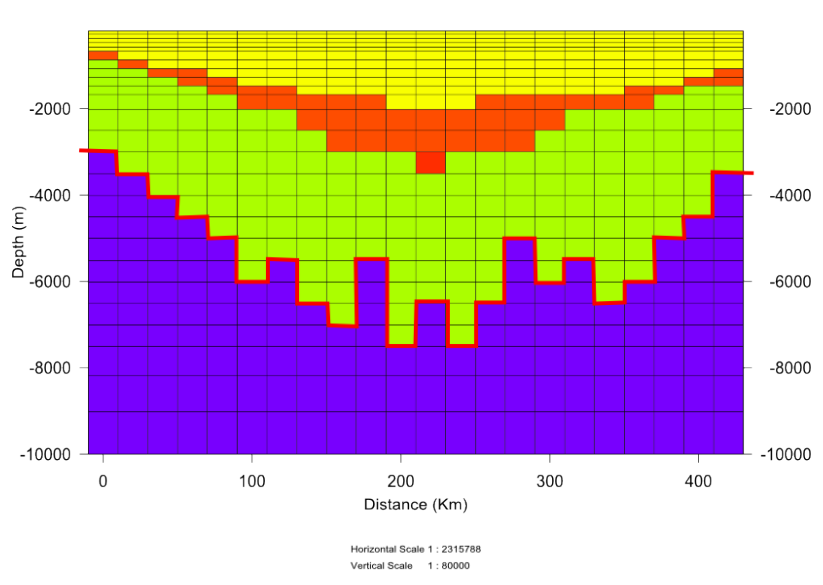

Figure 4: Seção MT1D composta sintética. A linha em vermelho marca o contraste de resistividade entre embasamento e bacia.

Um conjunto de 22 estações com espaçamento de $5 \mathrm{~km}$ foram distribuídas ao longo da seção. Curvas sintéticas de resistividades e fase foram geradas pelo algoritmo $2 \mathrm{D}$ de Rodi and Mackie (2001) baseadas no modelo geofísico proposto (Fig. 5) (Geosystem, 2011). A estação MT12 localiza-se no meio do perfil em um alto estrutural.

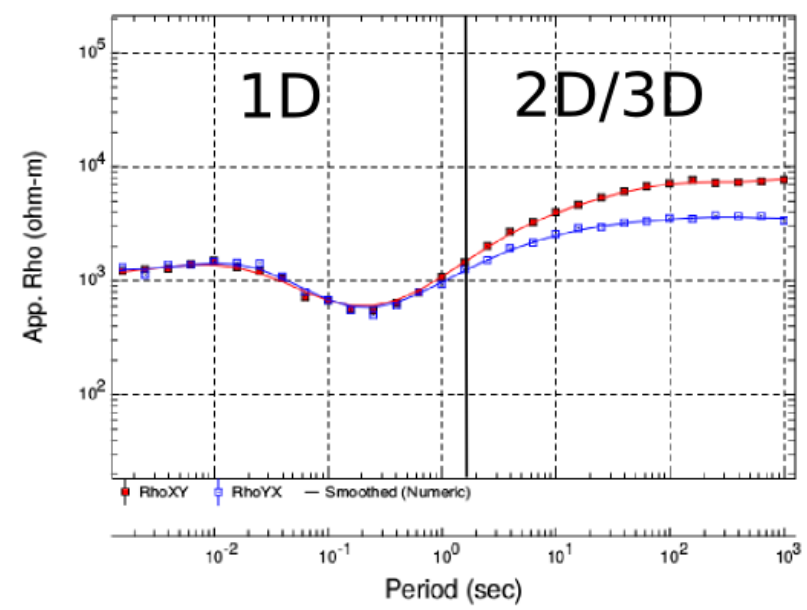

Figure 5: Dado sintético da estação MT12 criado para o experimento.

Considera-se um comportamento unidimensional nos dados sintéticos quando as curvas de resistividade aparente nas direções $X Y$ e $Y X$ estão sobrepostas, portanto sem variação lateral da resistividade. Descartouse a parte dos dados que não apresentava realidade 1D. Por exemplo, 2D e 3D (Fiona and Bahr, 2005). Esse 
corte representa a interface limítrofe do embasamento e da bacia sedimentar.

Inverteu-se através da utilização do algorítimo de 1D de Occam (Constable et al., 1987) a resistividade $X Y$ do dados sintético de cada estação MT que serviu de modelo inicial para topo e base do modelo de resistividade de camadas 1D (Meju, 1994). A Fig. 6 exemplifica a inversão 1D de Occam bem como modelo de camadas.
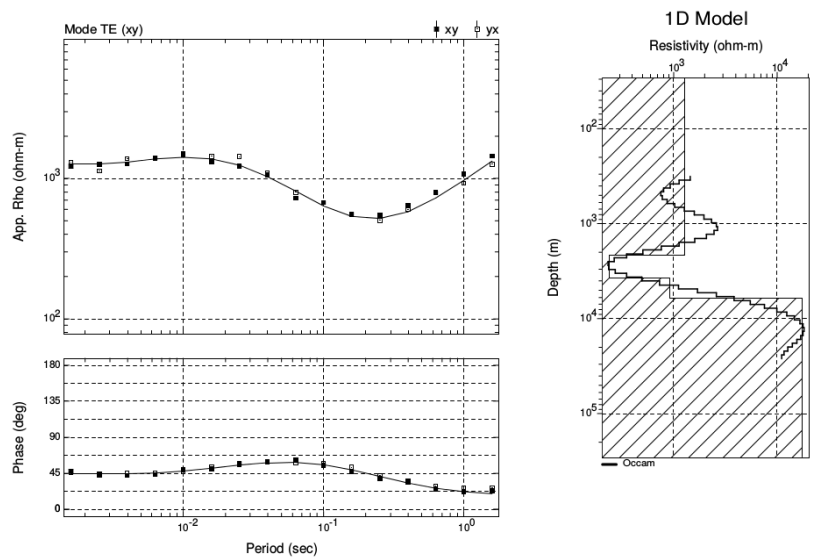

Figure 6: Exemplo do resultado da inversão do dado sintético da estação MT12. No painel a esquerda, valores de resistividade aparente e fase do dado observado. No painel a direita o resultado da inversão 1D de Occam e o modelo de camadas.

A Fig. 7 mostra o comparativo do resultado da seção composta MT 1D sintética com a superfície que delimita o embasamento mostrada na Fig. 4. O resultado evidencia boa correlação entre a seção composta MT 1D sintética e o embasamento, contudo as estações MT1, MT21 e MT22 próximas as bordas leste e oeste do modelo apresentaram erros na profundidade do embasamento da ordem de 500 metros.

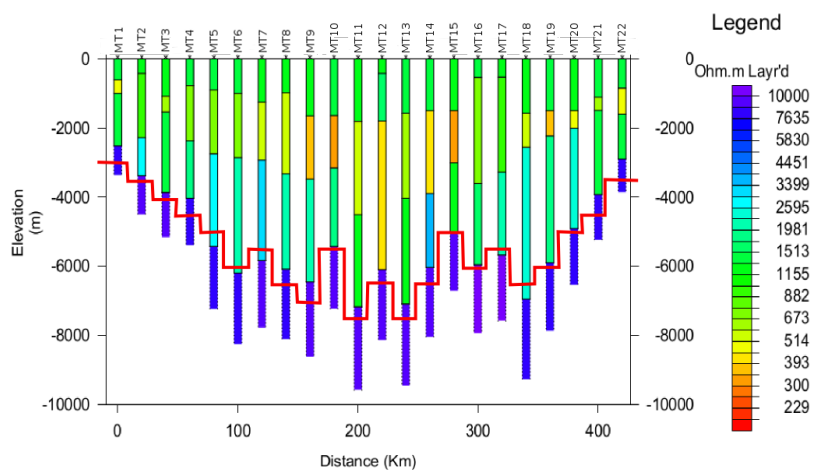

Figure 7: Seção MT1D composta sintética. A linha em vermelho representa o embasamento criado pelo modelo de resistividades sintético (Fig. 4). Os traços abaixo de cada estação representam os modelos de camadas que foram calculados pelas inversões 1D (Fig. 6).

O teste de hipótese proposto para a discretização da superfície do embasamento utilizando modelagem e inversão $1 \mathrm{D}$ foi capaz de delimitar uma superfície aproximada de alto contraste de resistividade que simularam o contraste de rochas entre sedimento e embasamento.

\section{Dado Real}

Os dados MT foram processados empregando a técnica robusta e referência remota (Gamble et al., 1979; Egbert and Booker, 1986) 150 estações MT espaçadas de $2 \mathrm{Km}$ ao longo do perfil de estudo foram utilizados na inversão 1D.

A mesma metodologia utilizada nos dados sintéticos foi utilizada nos dados reais (corte 1D e inversão de dados 1D de Occam) conforme mostrado na Fig. 6 (Constable et al., 1987).

A profundidade do embasamento foi determinada com a criação da seção MT 1D composta, baseada no contraste de resistividade entre o embasamento e a bacia sedimentar. A Fig. 8 mostra o resultado final das inversões 1D dos dados MT.

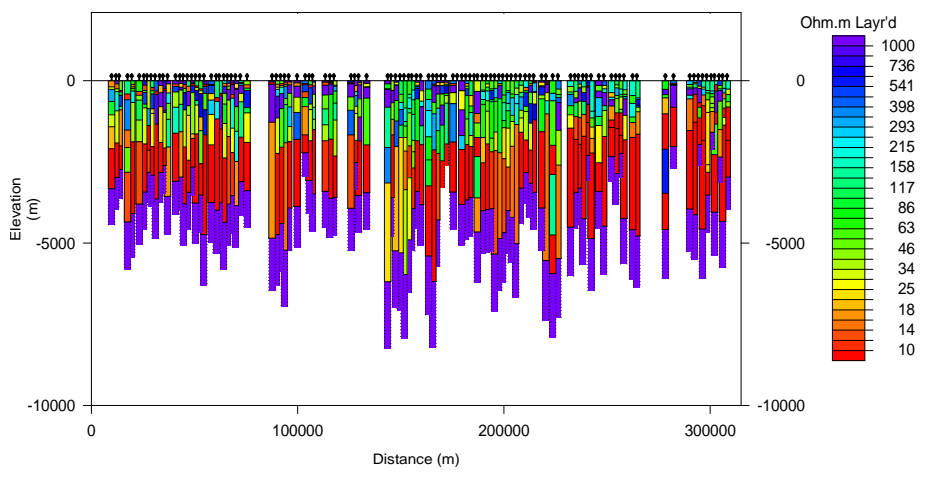

Figure 8: Seção composta 1D MT.

$\mathrm{Na}$ Tab. 1 são apresentados os parâmetros que foram utilizados na inversão 1D Occam. Salienta-se que o dado calculado convergia com 10 iterações para um número de camadas com valor de resistividade aparente de 45.

Table 1: Parâmetros do modelo inversão Occam

\begin{tabular}{|c|c|}
\hline Modo & TE \\
\hline Camadas & 45 \\
\hline Número de iterações & 10 \\
\hline
\end{tabular}

A Tab. 2 apresenta as características utilizadas na criação do modelo de camadas $1 \mathrm{D}$. O número alto de iterações garantiu a convergência do dado, cujo valor de RMS calculado foi 1 Ohm.m . O número de camadas definidas inicialmente como sendo 8 para cada inversão não foi utilizado para a interpretação de camadas sedimentares. Foi utilizada somente uma camada guia 
que se caracterizava pelo alto contraste de resistividade aparente em cada inversão.

Table 2: Parâmetros do modelo 1D de camadas

\begin{tabular}{|c|c|}
\hline Estações & 150 \\
\hline Modo & TE \\
\hline Camadas & 8 \\
\hline R.M.S & 1.0 \\
\hline Número de iterações & 100 \\
\hline
\end{tabular}

O modelo MT1D composto encontrado apresenta duas regiões com depocentros marcantes. O primeiro conjunto formado por 11 estações apresentou uma profundidade média de $5,5 \mathrm{Km}$. E um segundo conjunto de estações formado por 5 estações apresentou uma profundidade média de $5 \mathrm{Km}$ aproximadamente.

Já a região SE do modelo apresentou um conjunto de 6 estações que indicam uma profundade de embasamento de $4,2 \mathrm{Km}$.

A região NW do perfil apresenta aproximadamente um conjunto de 30 estações que em média apresentam valores de profundidade na ordem de $3,5 \mathrm{Km}$.

\section{Conclusões}

A seção Mt1D real indica um valor de depocentro de 5,5Km na região central do perfil. Os resultados obtidos para as regiões NW e SE do perfil são mais rasas atingindo uma média de $3,5 \mathrm{Km}$ na parte NW e $4,2 \mathrm{Km}$ na parte SE (Fig. 9). Tais resultados são coerentes com os dados de poços públicos e com as linhas de isópacas para a Bacia (Zalan and Wolf, 1987; Milani and Ramos, 1998; Milani and Zalan, 1999).

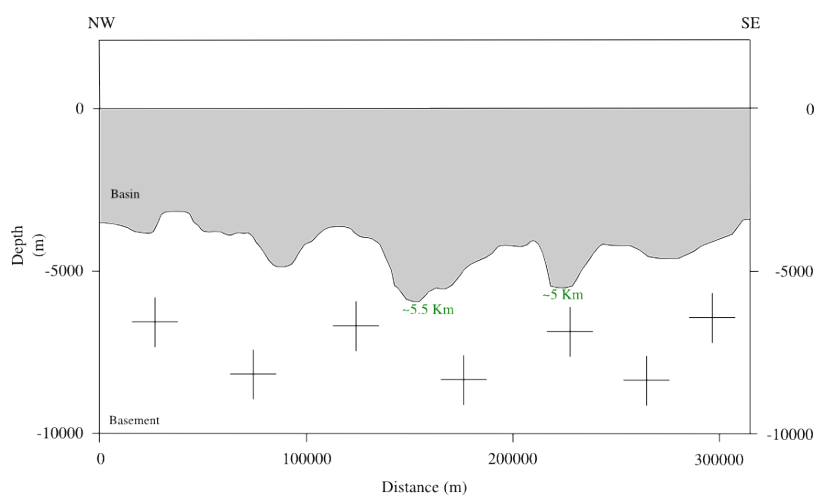

Figure 9: Embasamento Interpretado.

Grandes derrames como registrados pela Formação Serra Geral (Fig. 1) não comprometeram a estimativa da profundidade do embasamento como verificado na parte SE do perfil (Fig. 8).

O teste de hipóteses indica que o erro na previsão do relevo do embasamento aumenta nas bordas da seção 1D composta sintética (Fig. 7).

Em estações aonde foram registrados soleiras (Fig. 1) o resultado invertido para profundidade fica comprometido como é observado da parte SE da seção MT1D composta
(Fig. 8).

\section{Agradecimentos}

Os autores agradecem à ANP por ter cedido os dados, ao Observatório Nacional pelo fornecimento das instalações de pesquisa. E, Victor Ribeiro Carreira agradece ao CNPq pela concessão bolsa de mestrado, e o Dr. Sergio Luiz Fontes pela concessão da bolsa de Produtividade em Pesquisa. 


\section{References}

Árnason, K., 2008, The magneto-thelluric static shift problem: ISOR - Iceland GeoSurvey, Reykjavik, 17.

Bizzi, A. L., C. Schobenhaus, R. M. Vidotti, and Gonçalves, 2003, Geologia, tectônica e recursos minerais do Brasil: texto, mapas e SIG, 1 ed.: CPRM - Serviço Geológico do Brasil.

Borghi, L., 2002, A Bacia do Paraná: Anuário do Instituto de Geociências - IGEO, Departamentno de Geologia.

Constable, S. C., R. L. Parker, and C. G. Constable, 1987, Occam's inversion: A pratical algorithm for generating smooth models from eletromagnetic sounding data.: Geophysics, 52, 289-300.

Cordani, G., B. Neves, and R. Fuck, 1984, Estudo preliminar de integração do pré-cambriano com os eventos tectônicos da bacias sedimentares brasileiras: Revista Ciência Técnica do Petróleo, 27, 70p.

Egbert, G. D., and J. R. Booker, 1986, Robust estimation of geomagnetic transfer functions: Geophys. J. Royal Astron. Soc., 87, 173-194.

Figueiredo, I., 1997, Investigação magnetotelúrica nas serras do sudeste brasileiro (RJ/MG): Uma proposta de modelo crustal: Doutorado, Observatório Nacional, São Cristóvão, Rio de janeiro.

- , 2008, Heterogeneous crust and upper mantle across the SE Brazilian Highlands and the relationship to surface deformation as inferred from magnetotelluric imaging: Journal of Geophysical Research, 113.

Fiona, F., and K. Bahr, 2005, Pratical Magnetotellurics, $1^{a}$ ed.: Cambridge University Press.

Gamble, T., W. Goubau, and J. Clarke, 1979, Magnetotellurics with a remote magnetic reference: Electronics Research Center, University of Texas at Austin, volume 44 of Technical report.

Geosystem, S., 2011, Winglink user's guide, release 2.20.12. Printed in Milan, August 30, 2011.

Hawkesworth, C., K. Gallagher, L. Kirstein, M. Mantovani, D. Peate, and S. Turner, 2000, Tectonic controls on magmatism associated with continental break-up an example from the Paraná Etendeka Province: Earth and Planetary Science Letters, 179, 335-349.

Jones, A. G., 1988, Static shift of magnetotelluric data and its removal in a sedimentary basin environment: Geophysics, 53, 967-978.

Meju, M. A., 1994, Geophysical data analysis., 1 ed. ed.: Society of Exploration Geophysicists, 6.

Milani, E., A. Spadini, G. Terra, E. Silva, and G. Bueno, 2007, Boletim de geociências da Petrobras: Cenpes, v. 15.

Milani, E., and P. Zalan, 1999, An outline of the geology and petroleum systems of the Paleozoic interior basins of South America: Episodes, 22, 199-205.

Milani, E. J., and V. A. Ramos, 1998, Orogenias paleozóicas no domínio sul-ocidental do Gondwana e os ciclos de subsidência da Bacia do Paraná: Brazilian Journal of Geology, 28, 473-484.

Mwakirani, R., C. Simiyu, and J. Gichira, 2012, Application of transient electromagnetics in static shift correction for magnetotellurics data case study: Paka geothermal prospect in kenya: GRC - Transactions, 36, 1013-1016.

Rodi, W., and R. Mackie, 2001, Nonlinear conjugate gradients algorithm for 2-d magnetotelluric inversion: Geophysics, 66, 174-187.

Sternberg, B., J. Washburne, and L. Pellerin, 1988,
Correction for the static shift in magnetotellurics using transient electromagnetic soundings: Geophysics, 53.

Vail, P. R., R. M. Mitchum, and S. Thompson, 1977, Seismic stratigraphy and global changes of sea level: APPG. Seismic stratigraphy: applications to hydrocarbon exploration.

Vidotti, R., C. Ebinger, and J. Fairhead, 1998, Gravity signature of the western Paraná basin, Brazil: Earth and Planetary Science Letters, 117-132.

Vozoff, K., 1986, Magnetotelluric methods: Society of Exploration Geophysicists. Geophysics reprint series.

Word, D., H. Smith, and F. Bostick, 1970, An investigation of the magnetotelluric tensor impedance method: Society of Exploration Geophysicists. Technical report.

Zalan, P. V., and S. Wolf, 1987, Tectônica e sedimentação da Bacia do Paraná: Simpósio sul-brasileiro de geologia, SBG, 3, Atas, Curitiba-PR., 441-477. 